\title{
Correlations between neutrophil to lymphocyte ratio, blood eosinophils and clinical characteristics in chronic obstructive pulmonary disease
}

PNEUMOLOGY

1) Department of Pneumology, Iuliu Hatieganu University of Medicine and Pharmacy, Cluj-Napoca, Romania

2) "Leon Daniello" Clinical Hospital of Pneumology, Cluj-Napoca, Romania

DOI: $10.15386 / \mathrm{mpr}-1412$

Manuscript received: 01.07.2019 Received in revised form: 29.07.2019 Accepted: 11.08.2019

Address for correspondence: anna_f_rebrean@yahoo.com

This work is licensed under a Creative Commons Attribution-NonCommercialNoDerivatives 4.0 International License

Ana Florica Chis ${ }^{1,2}$, Carmen Monica Pop ${ }^{1,2}$

\begin{abstract}
Background and aims. Chronic obstructive pulmonary disease (COPD) is currently the third leading cause of death worldwide, with increasing mortality and morbidity. The neutrophil to lymphocyte ratio (NLR) and blood eosinophils level (EOS) represent biomarkers of inflammation in various diseases, with current research in the field of COPD. The aim of this study was to determine correlations of NLR and EOS with certain characteristics of COPD in a group of patients without major comorbidities.
\end{abstract}

Methods. We conducted an observational study on COPD patients admitted to the Clinical Hospital of Pneumology in Cluj-Napoca, Romania. The smoking history, body mass index (BMI), NLR, EOS, erythrocyte sedimentation rate (ESR), C-reactive protein (CRP) and the arterial partial pressure of oxygen $\left(\mathrm{PaO}_{2}\right)$ were determined. Functional assessment consisted of spirometric and BODE index determinations. The duration of hospitalization was expressed as the length of stay (LOS). The patients were divided into 3 subgroups: active smokers (AS), former smokers (FS) and never smokers (NS).

Results. No significant differences between AS and FS were found when age, airway obstruction, BODE index, $\mathrm{PaO}_{2}$, ESR and CRP were considered. The NLR was higher in AS versus FS ( $p=0.035)$, while EOS was lower in AS group ( $p=0.061)$. COPD patients with $\geq 300 \mathrm{EOS} / \mu \mathrm{L}$ had lower CRP, ESR levels and NLR compared to those with eosinophilia $<300 / \mu \mathrm{L}$ ( $\mathrm{p}=0.020, \mathrm{p}=0.009$ and $\mathrm{p}=0.007$, respectively). With a threshold of 3.5 for NLR, patients with lower NLR had lower CRP values $(\mathrm{p}=0.05)$. COPD patients with higher NLR had significant lower EOS levels $(p=0.018)$. Overall, the NLR and EOS were not correlated with the investigated characteristics $(p>0.05)$, but intragroup analysis (based on smoking status) revealed correlations with ESR $(\mathrm{p}=0.0001), \mathrm{CRP}(\mathrm{p}=0.053), \mathrm{BODE}$ index $(\mathrm{p}=0.029)$ and LOS $(\mathrm{p}=0.042)$.

Conclusions. AS have higher NLR and lower EOS levels versus FS. COPD patients with higher EOS level have lower CRP, ESR and NLR. In AS, EOS level is positively correlated with BODE index and negatively correlated with NLR.

Keywords: chronic obstructive pulmonary disease, neutrophils, lymphocyte, eosinophils, smoking

\section{Introduction}

According to Global Burden Disease, Chronic Obstructive Pulmonary Disease (COPD) claimed 2.93 million deaths in 2016 [1], and in 15 years it is predicted to become the leading cause of death worldwide [2]. The disease is characterized by progressive airflow limitation, that is not fully reversible, and is usually caused by significant exposure to noxious particles and gases [3]. The pathogenesis of COPD is not fully elucidated, and over the past years, research was based on identifying biomarkers that will provide information about the classification of the disease, the response to treatment or the future exacerbations risk $[4,5]$. There is a current growing interest in determining the inflammatory profile and clinical outcome in COPD patients by using simple, cost-effective blood tests, with interesting results on neutrophilto-lymphocyte ratio (NLR) and blood eosinophils level (EOS) [6-8]. The various 
limitations of these studies, however, contribute to many unanswered questions regarding these biomarkers.

The exposure to noxious particles, including cigarette smoke, induces activation of neutrophils. The consequence is the release of proteinases like neutrophil elastase or myeloperoxidase that cause the destruction of lung parenchyma and the release of mediators that contribute to airway inflammation and exessive mucus secretion $[9,10]$. Both tissue destruction and airway inflammation contribute to airflow limitation. The NLR is determined in peripheral blood and represents the ratio of neutrophils (NEU) and lymphocytes (LYM). The NLR is a rapid, easy and low-cost method and previous studies have proven its utility as a marker of inflammation in various conditions [11]. In COPD, an association between NLR and the severity of the disease was found [12], and several studies found higher values of NLR in acute exacerbations of COPD [13]. Over the past three years, NLR in COPD was studied as a marker of the severity of the disease or as a tool in predicting future exacerbations. However, the association of NLR with clinical and paraclinical characteristics and also clinical outcome in COPD patients is not fully elucidated.

Acute exacerbations of COPD have major implications on the severity of the disease, the prognosis, but also represent a financial burden for the healthcare system. Present studies on COPD patients aim to establish biomarkers that will predict and decrease the risk for future exacerbations, yet with conflicting results. Elevated blood EOS counts are found in a large variety of disorders, and there is an increasing interest in studying the role of EOS in COPD, as biomarkers of exacerbations, phenotypes, response to treatment and predictive tools. The levels of eosinophils in sputum are correlated with the blood eosinophil count [14], suggesting that peripheral blood measurements could serve as a more accessible biomarker of eosinophilic airway inflammation. The relationship between blood eosinophil count and clinical characteristics in COPD and the utility of EOS as predictors for future exacerbations represents a matter of constant actual concern.

The aim of our study was to find correlations between NLR, EOS and certain clinical and paraclinical characteristics of COPD patients, and also to investigate the potential role of these two biomarkers in assessing the clinical outcome.

\section{Material and methods}

\section{Subjects and selection criteria}

Sixty COPD patients from the Transylvania region of Romania, admitted to the "Leon Daniello" Clinical Hospital of Pneumology, Cluj-Napoca, Romania, were recruited in our study. The diagnosis and classification of COPD was based on 2017 Global Initiative for Obstructive Lung Disease (GOLD) guideline [3]. The inclusion criteria for the COPD group were: age $=41-89$ years, Romanian origin from the Transylvania region, written informed consent, previous diagnosis of COPD (at least 5 years). The exclusion criteria were: age $<40$ years or $>90$ years, previous diagnosis of: asthma, alpha 1 antitrypsin deficiency, interstitial lung disease, allergy, pneumonia, obstructive sleep apnea syndrome, acute coronary heart disease, chronic obstructive arteriopathy of the lower limbs, lower limb injuries, oral anticoagulant therapy, recent revascularization, thoracic surgery, acute renal failure, neoplasia, autoimmune diseases. Age, gender, body mass index (BMI) were determined.

BODE index, a predictor of survival in COPD patients, was determined for each patient, using the BMI, dyspnea (quantified with Modified Medical Research Council Dyspnea Scale - mMRC), airflow obstruction using Forced Expiratory Volume in $1^{\text {st }}$ second (FEV1s) and exercise capacity - using the distance (in meters) in the six-minute walk test (6MWT).

Length of Stay (LOS) was expressed in days of hospitalization. Smoking status was evaluated as: active smoker (AS), non-smokers (NS) and former smokers (FS), and the history of smoking was defined as pack-year index. The smoking abstinence period for FS was expressed as years without smoking (YWS).

\section{Peripheral blood measurements}

Five milliliters of venous blood were collected in fasting conditions from the antecubital vein, on the first day of admission in vacutainer tubes without anticoagulant. The blood was further centrifuged for $15 \mathrm{~min}$ at $4000 \mathrm{rpm}$ at room temperature $\left(20-25^{\circ} \mathrm{C}\right)$ and NLR, EOS, erythrocyte sedimentation rate (ESR), C-reactive protein (CRP) were determined. The arterial partial pressure of oxygen $\left(\mathrm{PaO}_{2}\right)$ was determined by peripheral arterial puncture, in sitting position, breathing room air, early in the morning, and analyzed with Astrup method.

\section{Functional tests}

Spirometry was performed according to the American Thoracic Society (ATS) and European Respiratory Society (ERS) guidelines [15], using a Modular Jaeger Vyntus ${ }^{\mathrm{TM}}$ Pneumo Spirometer (V-171416PAC, Serial No. 561439). The device consists in a pneumotach handle connected to a computer with a software that allows the technician to enter the patient's data, calibrate the flow sensor, perform the tests, and asses the results (flow-volume curve, parameters such as: Forced Vital Capacity, Tiffeneau Index, FEV1s, Peak Expiratory Flow). The COPD patients all had a value of the Tiffeneau Index lower than 0.7, and FEV1s was determined and expressed in percent of the predicted value [16] after inhalatory administration of $400 \mu \mathrm{g}$ Salbutamol, in order to appreciate the severity of the disease. The 6MWT was performed in accordance with ATS standard protocol [17]. 


\section{Statistical analysis}

Data were expressed as mean \pm standard deviation (SD). The statistical analyses were performed using IBM Statistical Package Program for the Social Sciences version 20. The significance of differences between groups and demographics was examined with the Student's t-test. Correlations between parameters were investigated with chi square test. A p value lower than 0.05 was considered as statistically significant.

\section{Ethical issues}

Our study received the approval from the Local Research Ethics Comittee of Iuliu Hatieganu University of Medicine and Pharmacy, Cluj-Napoca, no.298/29.06.2016. Written informed consent has been obtained from the patients. The study was conducted under the tenets of the Helsinki Declaration.

\section{Results}

Patients characteristics are presented in Table I.

Table I. Demographic and general characteristics of the study population.

\begin{tabular}{l|c|c} 
Characteristic & Mean & SD \\
\hline Age (years) & 66.25 & 9.36 \\
BMI & 29.46 & 6.30 \\
PYI & 29.52 & 18.29 \\
YWS (years) & 4.97 & 7.28 \\
FEV s (\% PV) & 44.58 & 15.78 \\
BODE index & 3.63 & 2.47 \\
PaO (mmHg) & 70.57 & 18.69 \\
NLR & 3.78 & 2.44 \\
ESR 1h & 23.26 & 24.42 \\
CRP (mg/dL) & 17.14 & 26.81 \\
LOS (days) & 9.50 & 4.48 \\
EOS (number/ $\boldsymbol{\mu L}$ ) & 212.50 & 168.51
\end{tabular}

Study population $=60$ COPD patients. SD - Standard Deviation; BMI - Body Mass Index; PYI - pack year index; YWS - years without smoking; FEV $\mathrm{s}$ (\% PV) - Forced Expiratory Volume in 1st second expressed as percent of the predicted value (PV); $\mathrm{PaO}_{2}$ - arterial blood oxygen partial pressure; NLR - Neutrophil to lymphocyte ratio; ESR - Erithrocyte Sedimentation Rate; CRP C-reactive protein; LOS - lenght of stay.
The 60 COPD patients included in our study were divided in three subgroups, based on the smoking status: active smokers (AS), $n=20$ - with a pack-year index greater than 10 , former smokers (FS), $n=28$ - patients with a history of smoking cessation longer than 5 years, and never smokers (NS), $n=12$ - patients that smoked less than 100 cigarettes during their lifetime.

We first investigated the characteristics between these subgroups of COPD patients. No statistically significant differences were observed between the AS and the FS when age, $\mathrm{FEV}_{1} \mathrm{~s}$, BODE index, $\mathrm{PaO}_{2}$, ESR or CRP were considered. The AS group had significantly lower BMI than the FS group, with a $p$ value $=0.047$. The NLR was higher in the AS group versus the FS group $(\mathrm{p}=0.035)$. Also, a borderline significance [18] was observed between the EOS levels of smokers versus nonsmokers $(\mathrm{p}=0.061)$, AS having lower EOS levels than FS, with a difference of $86.28 / \mu \mathrm{L}$.

The second purpose of our study was to investigate the EOS, CRP, ESR levels and NLR. Based upon the blood EOS level, the participants were divided into two subgroups: COPD patients with $\geq 300 \mathrm{EOS} / \mu \mathrm{L}$, and COPD patients with $<300 \mathrm{EOS} / \mu \mathrm{L}$. Patients in first group had lower CRP level $(p=0.020)$, lower ESR ( $p=0.009)$, and also lower NLR $(p=0.007)$. When a cut-off of $200 \mathrm{EOS} / \mu \mathrm{L}$ was used, the only statistically significant difference between groups was observed for NLR ( $\mathrm{p}=0.004)$. When a cut-off of 3.5 for NLR was considered, statistically significant differences were observed between the subgroups: COPD patients with lower NLR were younger $(\mathrm{p}=0.03)$ and had lower CRP values $(\mathrm{p}=0.05)$.

Furthermore, correlations between age, BMI, PYI, YWS, FEV1s, BODE index, PaO2, NLR, ESR, CRP, LOS and EOS were investigated, and overall correlations are presented in Table II. Positive correlations were found between age and NLR, age and ESR, smoking status, PYI and LOS ( $\mathrm{p}$ values in Table II). Negative correlations were discovered between smoking status and $\mathrm{PaO}_{2}$, smoking status and ESR, PYI and $\mathrm{PaO}_{2}, \mathrm{FEV}_{1} \mathrm{~s}$ and BODE index $(\mathrm{p}=0.0001)$, and EOS with NLR ( $p$ values in Table II). A borderline statistically significant negative correlation between CRP and $\mathrm{PaO}_{2}(p=0.061)$ was observed. No correlations were found between NLR or EOS and clinical/ paraclinical characteristics of the study population.

Table II. Correlations between COPD patients characteristics.

\begin{tabular}{|c|c|c|c|c|c|c|c|c|c|}
\hline & BMI & $\mathrm{FEV}_{1} \mathrm{~s}$ & BODE & $\mathrm{PaO}_{2}$ & NLR & ESR & CRP & LOS & EOS \\
\hline Age (years) & $0.266^{*}$ & $0.571^{*}$ & $0.857^{*}$ & $0.234 *$ & $0.027 * *$ & $0.020 * *$ & $0.176^{*}$ & $0.761 *$ & $0.401^{*}$ \\
\hline Smoking status & $0.164 *$ & $0.313^{*}$ & $0.745^{*}$ & $0.007 * * *$ & $0.288^{*}$ & $0.051 * * *$ & $0.117 *$ & $0.032 * *$ & $0.906^{*}$ \\
\hline PYI & $0.892 *$ & $0.574 *$ & $0.665^{*}$ & $0.002 * * *$ & $0.472 *$ & $0.114 *$ & $0.112 *$ & $0.038 * *$ & $0.634^{*}$ \\
\hline YWS (years) & $0.071^{*}$ & $0.170^{*}$ & $0.976^{*}$ & $0.412 *$ & $0.220^{*}$ & $0.598 *$ & $0.907 *$ & $0.220^{*}$ & $0.403^{*}$ \\
\hline n & 60 & 60 & 60 & 60 & 60 & 60 & 60 & 60 & 60 \\
\hline
\end{tabular}

$\mathrm{n}=$ number of patients; * - Pearson Correlation two tailed significance $\mathrm{p}$ value; $* *$ - $\mathrm{p}$ value with statistically significance, positive correlation; $* * *$ - $\mathrm{p}$ value with statistically significance, negative correlation; BMI - Body Mass Index; PYI - pack year index; YWS - years without smoking; FEV $\mathrm{s}(\% \mathrm{PV})$ - Forced Expiratory Volume in 1st second expressed as percent of the predicted value (PV); $\mathrm{PaO}_{2}$ - artherial blood oxygen partial pressure; NLR - Neutrophil to lymphocyte ratio; ESR - Erithrocyte Sedimentation Rate; CRP C-reactive protein; LOS - lenght of stay. 
Finally, intragroup analysis was performed in order to asses possible correlations for the AS, FS and NS groups of COPD patients. In the AS group, positive correlations were observed between NLR and age $(p=0.033)$, ESR and age (0.001), EOS and BODE index $(\mathrm{p}=0.029)$, BMI and $\mathrm{FEV}_{1} \mathrm{~s}(\mathrm{p}=0.013)$, and ESR and CRP $(\mathrm{p}=0.0001)$. Negative correlations were found between BMI and BODE index $(p=0.004), F V_{1} s$ and BODE index $(p=0.0001)$, BODE index and $\mathrm{PaO}_{2}(\mathrm{p}=0.006)$ and EOS and NLR $(\mathrm{p}=0.009)$.

In the FS group, the characteristics that had a positive correlation were: PYI and age (0.037), age and ESR $(p=0.014)$, CRP and YWS $(p=0.008)$, BODE index and CRP $(p=0.032)$, NLR and ESR $(p=0.011)$, NLR and LOS $(p=0.042)$, NLR and CRP ( $p=0.053$-borderline $)$, ESR and CRP $(p=0.0001)$, and ESR and LOS $(p=0.016)$.

When correlations in the NS group were assessed, no correlations between NLR or EOS and other characteristics were observed. BMI had a positive correlation with LOS $(\mathrm{p}=0.040)$ and a borderline negative correlation with ESR $(\mathrm{p}=0.058)$. In this subgroup, CRP correlated positively with ESR, and negatively with $\mathrm{PaO}_{2}(\mathrm{p}=0.0001$, and 0.052 respectively).

\section{Discussion}

COPD represents a major health problem worldwide, with increased costs for the healthcare system, especially for the management of acute exacerbations. The exacerbations of COPD are associated with higher mortality and a more rapid decline in health status [19]. Over the past three years, several biomarkers have been proposed as indicators of clinical outcome and survival prognosis, with interesting results for NLR and EOS, both parameters having the main advantages of being accessible, easy to determine, cost effective and repeatable.

In our study, the COPD patients had a severity stage of II, III and IV, according to the GOLD COPD classification [3] with $30 \%(n=18)$ of them presenting respiratory failure. The consequences of chronic hypoxemia, such as skeletal dysfunction and systemic inflammation reduce the quality of life in COPD patients and increase the risk of death [20]. The NLR represents a marker of systemic inflammation and has been widely investigated in different diseases, but its relationship with COPD has been studied only recently and is far from being completely understood. In our study population, the NLR had a mean value of $3.78 \pm 2.44$, lower than the previously reported values [6,21]. A possible explanation for this might be the fact that we used multiple exclusion criteria, many of the excluded diseases presenting with systemic inflammation that could increase the NLR. In an observational study published in 2016, Lee et al found that the NLR was significantly higher in COPD patients versus controls, and more important, it was correlated with the BMI, FEV $\mathrm{F}_{1} \mathrm{~s}$, BODE index, 6MWT and mMRC scale. Moreover, when a cut-off of 2.8 was considered, NLR represented an independent predictor with a borderline significance for respiratory hospitalisation [21]. The results of our research showed that in active and former smokers COPD patients, NLR correlates with age, PYI, ESR and CRP . Yao et al. (2017) found that in COPD patients, elevated NLR may be associated with long-term mortality [22]. The results of our study suggest that NLR is an important factor in evaluating the inflammatory status and hospitalization in smokers and ex-smokers, but not for the patients that developed COPD due to exposure to biomass and noxious particles, other than cigarette smoke. Furthermore, NLR might serve as a useful biomarker in determining the etiology of an exacerbation, by correlating it to other inflammatory biomarkers.

When smoking status was considered, a positive correlation between NLR and ESR was observed in the former smokers group, but not in the non-smokers and active smokers groups. These results might indicate that neutrophilic inflammation found in ex-smokers is linked to other inflammatory biomarkers, such as ESR, in highly selected patients. Cigarette smoke is incriminated in activating macrophages and epithelial cells in the airways, to release several mediators that neutrophils and lymphocytes into the lungs [23]. Gamble et al. (2007) found that even after smoking cessation, this inflammation persist [24], our results being consistent with these findings. Furthermore, in our population, the AS group had significantly higher values of NLR than the FS group, indicating that neutrophilia is much more important when smoking continues. A negative correlation was observed in our study between NLR and EOS in the AS and FS groups, indicating the existence of different phenotypes of inflammation, that might lead to different therapeutic approaches in COPD patients, as reported in previous studies $[25,26]$.

Higher blood EOS levels were observed in asthma and allergic patients, and recent research focuses on the importance of EOS in COPD as predictor of future exacerbations [27] and response to treatment, although some authors consider that one single biomarker to determine future exacerbations of the disease might not be enough [28]. The level of blood EOS in our patients was $212.5 \pm 168.51$ / $\mu \mathrm{L}$, with $13.33 \%$ patients having more than $300 \mathrm{EOS} / \mu \mathrm{L}$, thus revealing the presence of eosinophilic inflammation for a subgroup of COPD patients, in the absence of other risk factors. For this subgroup, the levels of CRP, NLR and ESR were significantly lower, suggesting that an eosinophilic phenotype could be defined, as previous reports stated [29]. The blood EOS levels were significantly lower in active smokers versus non-smokers or former smokers, suggesting that smoking might increase the recruitment or retention of eosinophils in specific sites, perhaps in distal lung areas [30], as recently reported by Martinez et al. (2018). Only for the active smokers group of our study, the patients with higher EOS levels had a greater BODE index, indicating a connection between these two parameters, both proposed as biomarkers of survival and mortality. 
The BMI in the active smokers group was significantly lower than the BMI in the former smokers group, similar to previous reports [31]. Although the BMI had a positive correlation with the LOS, patients with greater BMI had higher values of $\mathrm{FEV}_{1} \mathrm{~s}$ and lower BODE index, suggesting the "obesity-paradox" in COPD patients [32]. Our research also found positive correlations between PYI and LOS, indicating the importance of continuous smoking in clinical outcome of COPD patients.

\section{Conclusions}

In our group of COPD patients, we found no significant correlations between the NLR or EOS and clinical/functional/inflammation parameters. Considering smoking status, NLR and EOS might serve as useful biomarkers for inflammation (together with ESR and CRP), functional testing (BODE index) and clinical outcome (LOS) in COPD patients. Patients with higher blood EOS had lower NLR suggesting the presence of two distinct phenotypes of COPD patients. Active smokers have a significantly higher NLR and lower EOS versus former smokers. However, further larger studies are needed in order to assess the clinical importance of NLR and EOS.

\section{Acknowledgements}

This study is part of the Ana Florica Chis $\mathrm{PhD}$ research program: "Implications of inflammatory markers and IL-6 -597A/G and VEGF $+936 \mathrm{C} / \mathrm{T}$ genetic polymorphisms in the chronic obstructive pulmonary disease patient's profile", no. 7690/97/15.04.2016, funded through internal research programs by the Iuliu Hatieganu University of Medicine and Pharmacy, Cluj-Napoca, Romania.

\section{References}

1. GBD 2016 Causes of Death Collaborators. Global, regional, and national age-sex specific mortality for 264 causes of death, 1980-2016: a systematic analysis for the Global Burden of Disease Study 2016. Lancet. 2017;390:1151-1210.

2. Alwan A. Global Status Report on Non Communicable Diseases 2010. Geneva, Switzerland: World Health Organization, 2011.

3. Vogelmeier CF, Criner GJ, Martinez FJ, Anzueto A, Barnes PJ, Bourbeau J, et al. Global strategy for the diagnosis, management, and prevention of chronic obstructive lung disease 2017 report: GOLD executive summary. Eur Respir J. 2017;49.

4. Chen YW, Leung JM, Sin DD. A Systematic Review of Diagnostic Biomarkers of COPD Exacerbation. PLoS One. 2016;11:e0158843.

5. Fermont JM, Masconi KL, Jensen MT, Ferrari R, Di Lorenzo VAP, Marott JM, et al. Biomarkers and clinical outcomes in COPD: a systematic review and meta-analysis. Thorax. 2019;74:439-446.
6. Paliogiannis P, Fois AG, Sotgia S, Mangoni AA, Zinellu E, Pirina $\mathrm{P}$, et al. Neutrophil to lymphocyte ratio and clinical outcomes in COPD: recent evidence and future perspectives. Eur Respir Rev. 2018;27. pii:170113.

7. Teng F, Ye H, Xue T. Predictive value of neutrophil to lymphocyte ratio in patients with acute exacerbation of chronic obstructive pulmonary disease. PLoS One. 2018;13:e0204377.

8. Tsiligianni I, Kaplan AG. Are blood eosinophils a primetime biomarker for COPD management decisions?. Int $\mathrm{J}$ Chron Obstruct Pulmon Dis. 2018;13:1889-1891.

9. Bourdin A, Burgel PR, Chanez P, Garcia G, Perez T, Roche $\mathrm{N}$. Recent advances in COPD: pathophysiology, respiratory physiology and clinical aspects, including comorbidities. Eur Respir Rev. 2009;18:198-212.

10. Houghton AM, Rzymkiewicz DM, Ji H, Gregory AD, Egea EE, Metz HE, et al. Neutrophil elastase-mediated degradation of IRS-1 accelerates lung tumor growth. Nat Med. 2010;16:219-223.

11. Imtiaz F, Shafique K, Mirza SS, Ayoob Z, Vart P, Rao S. Neutrophil lymphocyte ratio as a measure of systemic inflammation in prevalent chronic diseases in Asian population. Int Arch Med. 2012;5:2.

12. Furutate R, Ishii T, Motegi T, Hattori K, Kusunoki Y, Gemma A, et al. The neutrophil to lymphocyte ratio is related to disease severity and exacerbation in patients with chronic obstructive pulmonary disease. Intern Med. 2016;55:223229.

13. Bilir B, Altintas N, Aydin M, Oran M, Özsu S, Tutar Ü. The predictive role of neutrophil to lymphocyte ratio in chronic obstructive pulmonary disease. Eur J Gen Med. 2016;13(2):105-110.

14. Bafadhel M, McKenna S, Terry S, Mistry V, Reid C, Haldar P, et al. Acute exacerbations of chronic obstructive pulmonary disease: identification of biologic clusters and their biomarkers. Am J Respir Crit Care Med. 2011;184:662671.

15. Pauwels RA, Buist AS, Calverley PM, Jenkins CR, Hurd SS; Gold Scientific Committee. Global strategy for the diagnosis, management, and prevention of chronic obstructive pulmonary disease. NHLBI/WHO Global Initiative for Chronic Obstructive Lung Disease (GOLD) Workshop summary. Am J Respir Crit Care Med. 2001;163:1256-1276.

16. Quanjer $\mathrm{PhH}$. Standardized lung function testing: report of the Working Party for the European Community for Steel and Coal. Bull Eur Physiopathol Respir. 1983;19(Suppl 5):22-27.

17. ATS Committee on Proficiency Standards for Clinical Pulmonary Function Laboratories. ATS statement: guidelines for the six-minute walk test. Am J Respir Crit Care Med. 2002;166:111-117.

18. Hackshaw A, Kirkwood A. Interpreting and reporting clinical trials with results of borderline significance. BMJ. 2011;343:d3340.

19. Suissa S, Dell'Aniello S, Ernst P. Long-term natural history of chronic obstructive pulmonary disease: severe exacerbations and mortality. Thorax. 2012;67:957-963. 
20. Kent BD, Mitchell PD, McNicholas WT. Hypoxemia in patients with COPD: cause, effects, and disease progression. Int J Chron Obstruct Pulmon Dis. 2011;6:199-208.

21. Lee SJ, Lee HR, Lee TW, Ju S, Lim S, Go SI, et al. Usefulness of neutrophil to lymphocyte ratio in patients with chronic obstructive pulmonary disease: a prospective observational study. Korean J Intern Med. 2016;31:891-898.

22. Yao C, Liu X, Tang Z. Prognostic role of neutrophillymphocyte ratio and platelet-lymphocyte ratio for hospital mortality in patients with AECOPD. Int $\mathrm{J}$ Chron Obstruct Pulmon Dis. 2017;12:2285-2290.

23. Barnes PJ. Inflammatory mechanisms in patients with chronic obstructive pulmonary disease. J Allergy Clin Immunol. 2016;138:16-27.

24. Gamble E, Grootendorst DC, Hattotuwa K, O'Shaughnessy T, Ram FS, Qiu Y. et al. Airway mucosal inflammation in COPD is similar in smokers and ex-smokers: a pooled analysis. Eur Respir J. 2007;30:467-471.

25. Acartürk Tunçay E, Karakurt Z, Aksoy E, Saltürk C, Gungor $\mathrm{S}$, Ciftaslan N, et al. Eosinophilic and non-eosinophilic COPD patients with chronic respiratory failure: neutrophilto-lymphocyte ratio as an exacerbation marker. Int J Chron Obstruct Pulmon Dis. 2017;12:3361-3370.

26. Aksoy E, Karakurt Z, Gungor S, Ocakli B, Ozmen I, Yildirim
E, et al. Neutrophil to lymphocyte ratio is a better indicator of COPD exacerbation severity in neutrophilic endotypes than eosinophilic endotypes. Int J Chron Obstruct Pulmon Dis. 2018;13:2721-2730.

27. Yun JH, Lamb A, Chase R, Singh D, Parker MM, Saferali A, et al. Blood eosinophil count thresholds and exacerbations in patients with chronic obstructive pulmonary disease. J Allergy Clin Immunol. 2018;141:2037-2047.e10.

28. Wedzicha JA. Eosinophils as biomarkers of chronic obstructive pulmonary disease exacerbation risk. Maybe Just for Some? Am J Respir Crit Care Med. 2016;193:937-938.

29. O'Donnell R, Breen D, Wilson S, Djukanovic R. Inflammatory cells in the airways in COPD. Thorax. 2006;61:448-454.

30. Martinez CH, Li SX, Hirzel AJ, Stolberg VR, Alexis NE, Barr RG, et al. Alveolar eosinophilia in current smokers with chronic obstructive pulmonary disease in the SPIROMICS cohort. J Allergy Clin Immunol. 2018;141:429-432.

31. Piirtola M, Jelenkovic A, Latvala A, Sund R, Honda C, Inui $\mathrm{F}$, et al. Association of current and former smoking with body mass index: A study of smoking discordant twin pairs from 21 twin cohorts. PLoS One. 2018;13:e0200140.

32. Franssen FM, O'Donnell DE, Goossens GH, Blaak EE, Schols AM. Obesity and the lung: 5. Obesity and COPD. Thorax. 2008;63:1110-1117. 